\title{
Four-Leg Transformer with Defined Zero Sequence Impedance for Ground Fault Compensation
}

\author{
Mohsen Rezaei \\ The Research and Innovation Centre \\ for Electrical Engineering (RICE) \\ University of West Bohemia \\ Plzen, Czech Republic \\ rezaei@fel.zcu.cz
}

\author{
Jakub Talla \\ The Research and Innovation Centre \\ for Electrical Engineering (RICE) \\ University of West Bohemia \\ Plzen, Czech Republic \\ talic@rice.zcu.cz \\ Zdenek Peroutka \\ The Research and Innovation Centre \\ for Electrical Engineering (RICE) \\ University of West Bohemia \\ Plzen, Czech Republic \\ pero@rice.zcu.cz
}

\author{
Tomas Komrska \\ The Research and Innovation Centre \\ for Electrical Engineering (RICE) \\ University of West Bohemia \\ Plzen, Czech Republic \\ komrska@rice.zcu.cz
}

\begin{abstract}
Single-phase ground faults are the most common faults in medium-voltage distribution grids. High-impedance grid grounding using a special four-leg transformer is discussed in the paper. The transformer's zero-sequence impedance differs from positive-sequence impedance and it is designed to compensate the earth faults currents in Medium Voltage (MV) distribution grids when single-phase ground fault occurs. The designed transformer can replace the traditional arc suppression coils (ASC) in the case where no distribution transformer neutral is available or distributed compensation is required. Alternatively, the transformer can operate in parallel with the ASC.
\end{abstract}

Keywords-Single Earth Fault, Distribution Grid, Petersen Coil, Zero Sequence Current, Transformer

\section{INTRODUCTION}

One of the most common faults in the distribution network is a single phase to ground fault [1]. Grounding has an important role in medium voltage grid. There are various forms of grounding such as isolated neutral, directly grounded neutral and high-impedance grounded grid. In resonant grounding, a tunable reactance is installed between the system's neutral and the earth. The reactance of the arc suppression coil ASC (Petersen coil [2]) is connected in parallel with the parasitic capacitance of the grid and both parameters build a resonant circuit. When the resonant frequency of the parallel circuit corresponds to the frequency of the grid (e.g. $50 \mathrm{~Hz}$ ), the fault current can be effectively compensated. [3] - [6]. A Petersen coil consists of an inductor dealing with ground faults by an inductive current which is in opposite to the grid capacitive current [7]. Coil tuning is timeconsuming procedure. Although the main problem is the resonant point. To achieve the most effective compensation, the resonant frequency of the parallel circuit coil - parasitic capacitance need to be exactly $50 \mathrm{~Hz}$. On the other hand, in this point the zero-sequence voltage is maximal, which is undesired; it leads to overvoltage, insulation stress, etc. Therefore, the coils are usually slightly under tuned, but it affects the residual current, and leads to higher uncompensated current component. As a result, distribution power grids, the ASC tuning is a tradeoff between maximum of the zero-sequence component and the residual current. [8] - [15]. When the single earth fault occurs, the three phase voltage will be asymmetric composing of both, the positive and the zero-sequence components [16-17]. Recently, researchers have prioritized the selection of appropriate simulation software to achieve useful results [18], hence in this paper Piece-wise Linear Electrical Circuit Simulation (PLECS) is so efficient software for designing the 4-leg transformer.

The zero sequence current can be controlled by 4-leg transformer. A mathematical model of a special transformer which is able to compensate earth fault in MV distribution grids is discussed in the paper. It is designed to generate compensation current, when zero-sequence component is presented in the grid. Impedance of the zero-sequence component is designed by fourth leg reluctance of the magnetic core, and zero-sequence impedance affects the size of the compensation current. The transformer is connected to phase conductors and to the earth. It can be installed either in the distribution substation or elsewhere in the grid. It can replaces the ASC or operates in parallel with the ASC. To verify the validity of the model, a simulation in MATLAB/PLECS is also presented. The structure of the article is as follows: After reviewing previous studies in Section I, in section II, a model for the distribution network is presented, and then in section III, the 4-leg transformer is introduced. Section IV deals with the simulation results using three types of compensation and at the end in Section $\mathrm{V}$, the conclusion is mentioned.

\section{MODEL OF NETWORK}

\section{A. Resonant grounded $M V$ distribution grids}

Fig. 1 shows the scheme of a MV distribution power grid $(22 \mathrm{kV} / 50 \mathrm{~Hz})$, grounded via an ASC. A distribution transformer $110 / 22 \mathrm{kV}$ supplies four feeders. After the ground fault current occurs, the faulty phase voltage drops to zero approximately and the voltage of other phases will increase to line-line voltage.

Fig. 2 shows the phasor diagram of voltage and current state of the grid and the fault is present in feeder 3. In this feeder after the single-phase ground fault, the phase $\mathrm{C}$ voltage is dropped to zero. As shown in Figure 2, the angle difference between currents $A$ and $B$ is 60 degrees. The sum of currents $\mathrm{A}$ and $\mathrm{B}$ is a capacitive current, and to overcome it, ASC must 
inject an inductive current in the opposite direction.

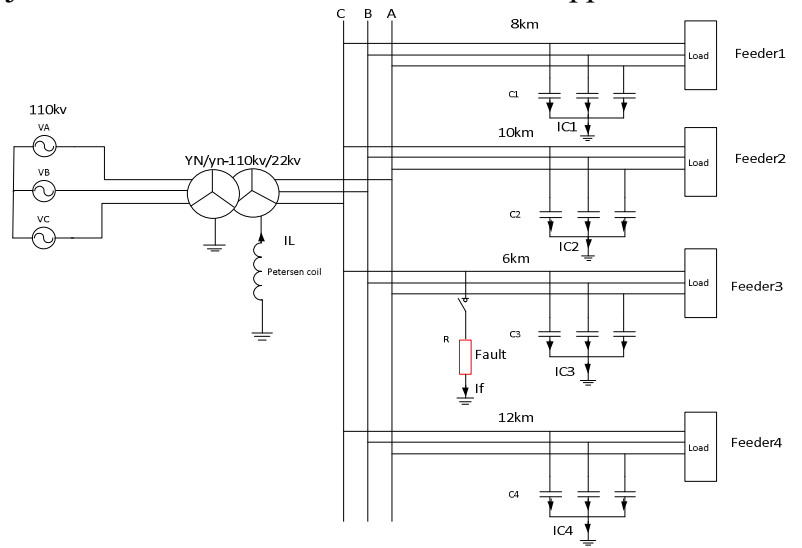

Fig. 1. Distribution grid with Petersen coil

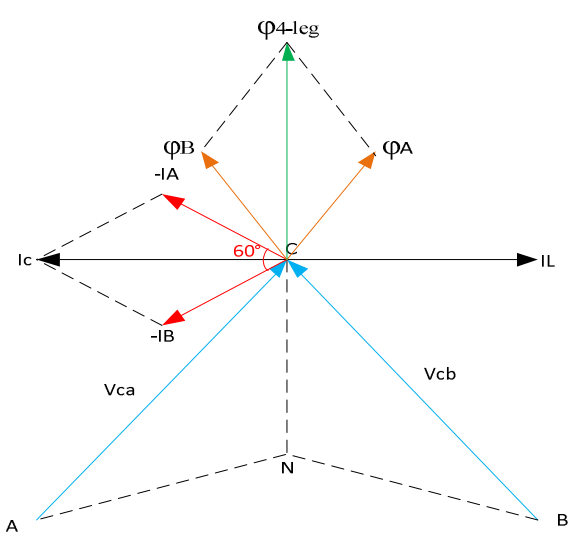

Fig. 2. Phasor diagram of current and voltages

As shown in Fig.2, total capacitive current flows through the fault spot when the grid is uncompensated. For better understanding, equivalent circuit of single-phase-earth fault on Peterson coil compensated system is depicted in Fig.3, where $R_{f}$ is the grounding fault resistance, $A S C$ is equivalent inductance of arc suppression coil and $L_{A S C}$, $C$ and $R$ are equivalent inductance, capacitance and resistance of normal line respectively.

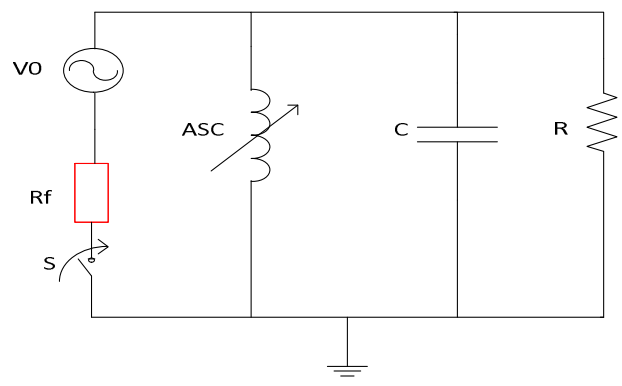

Fig. 3. Equivalent circuit for zero-sequence component modelling a single-phase earth fault in power grid compensated by a ASC

In normal operation, the inductance of Petersen coil should be matched with capacitors. Therefore the inductive current of ASC $\mathrm{I}_{\mathrm{L}}$ is equal to summation of entire zerosequence capacitive currents:

$$
\begin{gathered}
I_{L}=\sum I_{C} \\
L=\frac{1}{3 \omega^{2} \sum C}
\end{gathered}
$$

Where $I_{L}$ is ASC's current and $I_{C}$ is capacitive current. Via formula (2), value of ASC's inductance can be calculated, where $L$ and $\sum C$ are ASC's inductance and total capacitor of grid and $\omega=2 \pi f$ that $f$ is frequency of grid [19].

\section{4-LEG TRANSFORMER WITH DEFINED ZERO-SEQUENCE IMPEDANCE}

The proposed solution is to use a 4-leg transformer that this compensation system, unlike the ASC, can be installed in any place in the grid, and it is suitable for distributed compensation.

\section{A. 4-leg transformer}

Three phase transformer with 4-leg magnetic core is shown in Fig.4. The primary winding is star-connected and the star center is grounded. The ratio of this transformer is $22 \mathrm{kV} / 0.4 \mathrm{kV}$. In normal operation three-phase voltage is balanced $u a+u b+u c=0$ and there is no zero-sequence component is present and fluxes are also balanced $\varphi a+\varphi b+$ $\varphi c=0$ without flux in 4 th leg. Under fault condition: one voltage is low (zero in extreme case) and zero-sequence component is present $u a+u b+u c \neq 0$ and $\varphi a+\varphi b+\varphi c$ flows via the 4 th leg. To achieve effective fault current compensation, the air gap in the 4th leg of the transformer must be properly designed to achieve defined zero-sequence component impedance and thus the magnitude of the compensation current. Unlike the zero-sequence component, the three legs of the transformer with the winding are without air gap. The impedance of the transformer for the positive component is high. If the zero sequence voltage is presented, the three identical currents flow via primary phase a, b, c and the resulting compensation current is $i_{n}=i_{a}+i_{b}+i_{c}$. Relation between flux and voltage is mentioned by (4) where $V$ is phase voltage and $\varphi$ is flux.

$$
\bar{\varphi}_{4 t h-l e g}=\bar{\varphi}_{a}+\bar{\varphi}_{b}+\bar{\varphi}_{c}
$$

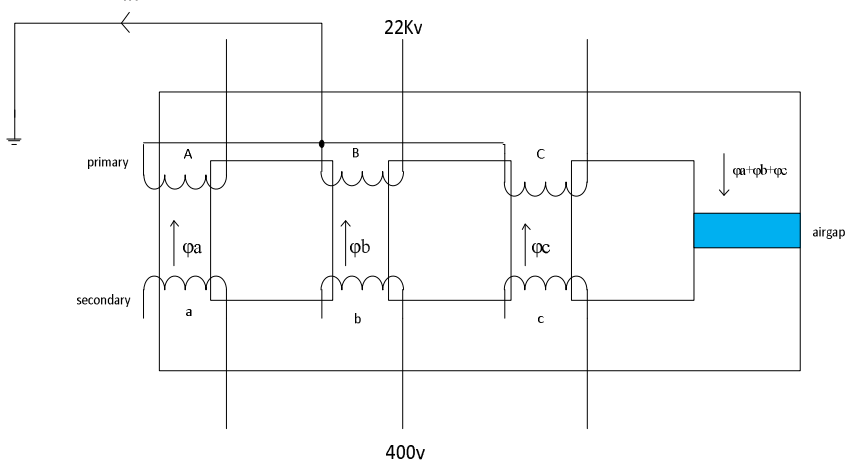

Fig. 4. 4-leg three phase transformer $22 \mathrm{kV} / 0.4 \mathrm{kV}$

$$
\varphi=\int V d t
$$

If single-phase fault occurs to ground, the voltage of that phase will be zero and as a result the flux passing through the 
fourth column of the transformer is equal to the sum of the fluxes of the other phases and the flux passing through is not zero.

\section{SIMULATION RESULTS}

In order to verify theoretical assumptions, a simulation model is built up in PLECS software. Fig.1 shows the simulation system with four feeders including three overhead lines and one cable line, a YN/yn transformer, a bus, and a ASC. The primary winding of 4-leg transformer will be connected to grid. Detailed parameters of the simulation system are shown in Table I.

TABLE I. THE PARAMETERS OF LINES

\begin{tabular}{|c|c|c|c|c|}
\hline $\begin{array}{c}\text { Type of } \\
\text { line }\end{array}$ & $\begin{array}{c}\text { Phase } \\
\text { sequence }\end{array}$ & $\mathbf{R}\left(\frac{\Omega}{k m}\right)$ & $\mathbf{L}\left(\frac{m H}{k m}\right)$ & $\mathrm{C}\left(\frac{\mu F}{k m}\right)$ \\
\hline \multirow{2}{*}{$\begin{array}{l}\text { Overhead } \\
\text { line }\end{array}$} & $\begin{array}{c}\text { positive } \\
\text { sequence }\end{array}$ & 0.17 & 1.21 & 0.0097 \\
\hline & $\begin{array}{c}\text { zero } \\
\text { sequence }\end{array}$ & 0.23 & 5.47 & 0.006 \\
\hline \multirow{2}{*}{ Cable line } & $\begin{array}{c}\text { positive } \\
\text { sequence }\end{array}$ & 0.27 & 0.26 & 0.34 \\
\hline & $\begin{array}{c}\text { zero } \\
\text { sequence }\end{array}$ & 2.70 & 1.02 & 0.28 \\
\hline
\end{tabular}

The earth fault in the phase $\mathrm{C}$ system frequency is $50 \mathrm{~Hz}$ and simulation duration is $0.4 \mathrm{~s}$. The earth fault in the phase $\mathrm{C}$ occurs in time $0.2 \mathrm{~s}$ in feeder 3 . Feeders 1,2 and 3 are overhead lines and feeder 4 is cable line. The lengths of feeder 1 is $8 \mathrm{~km}$, feeder 2 is $10 \mathrm{~km}$, feeder 3 is $6 \mathrm{~km}$ and feeder 4 is $12 \mathrm{~km}$ respectively. Through the simulation system, resistance's fault is $20 \Omega$.

\section{A. Network compensated by ASC}

The single-phase earth fault applied in time $0.2 \mathrm{~s}$ on phase $\mathrm{c}$ in feeder 3. Phase voltages are shown in Fig. 5. The voltage of phase $\mathrm{c}$ drops to zero while the voltage of other phases increases. The zero-sequence voltage component is presented and it causes that the current of the ASC increases that both of them are depicted in Fig.6 and as seen the current has dc component. Earth fault current, faulty feeder current and summation of current of healthy feeders are shown on Fig. 7, it is reduced to nearly $10 \mathrm{~A}$. The value of Petersen coil is calculated by (2) and is $1.16821 \mathrm{H}$ that is connected to neutral's secondary side of $110 \mathrm{kV} / 22 \mathrm{kV}$ transformer.

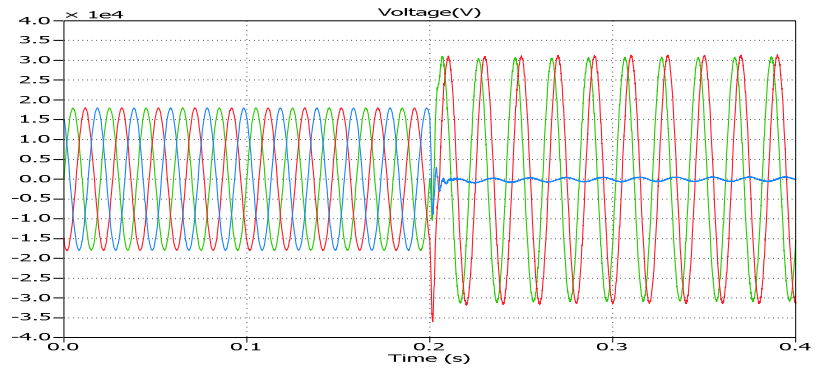

Fig. 5. Voltages of feeder 3 after single earth fault

In this case the compensation current is equal to current of ASC. Using ASC has several major problems, that some of them explained in [17]. In addition, the Petersen coil cannot be connected anywhere in the network, and in case the secondary winding of the distribution transformer is of delta connection, no neutral point is available for an ASC.

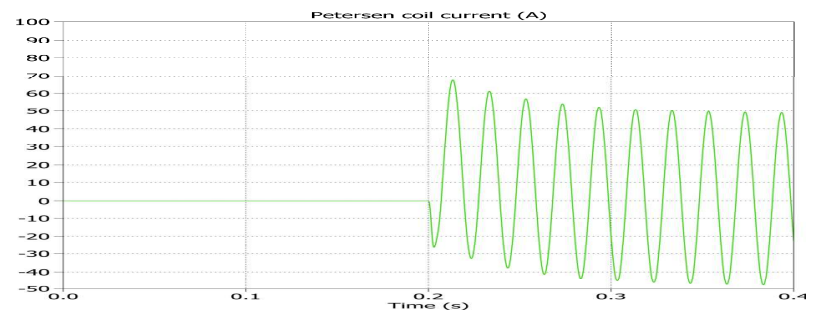

(a)

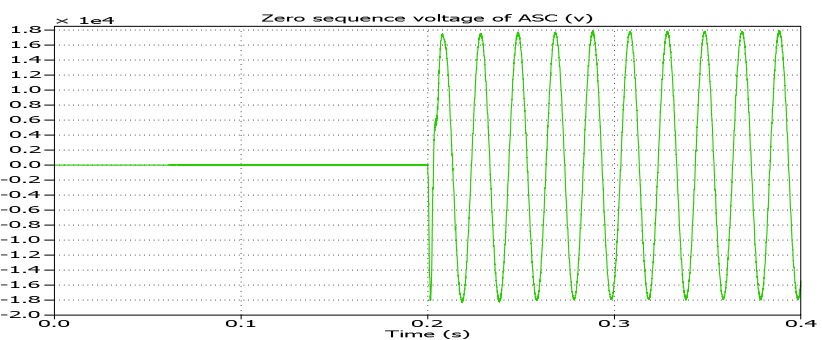

(b)

Fig. 6. (a) ASC's current. (b) Zero sequence voltage of ASC

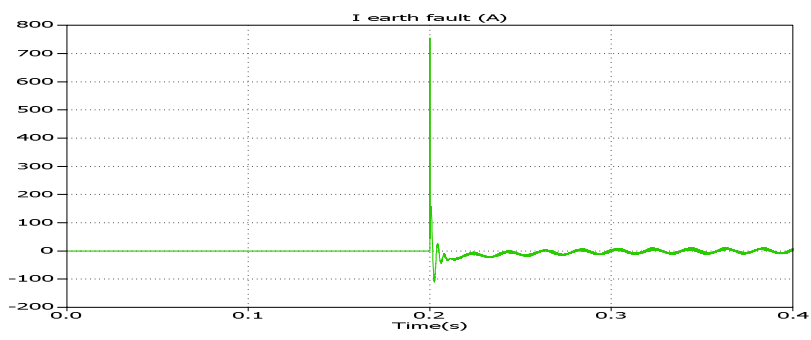

(a)

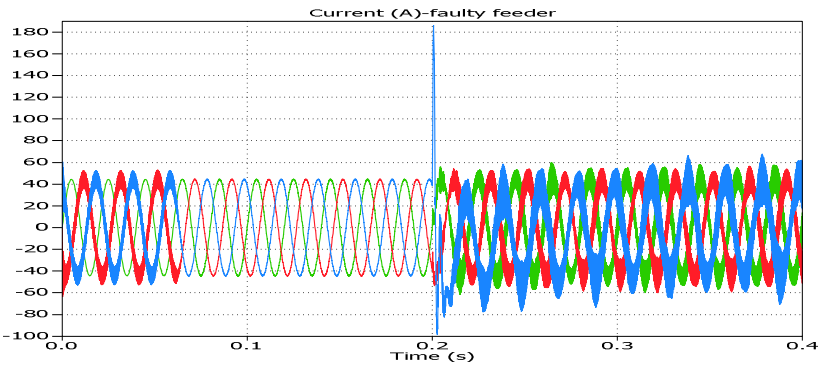

(b)

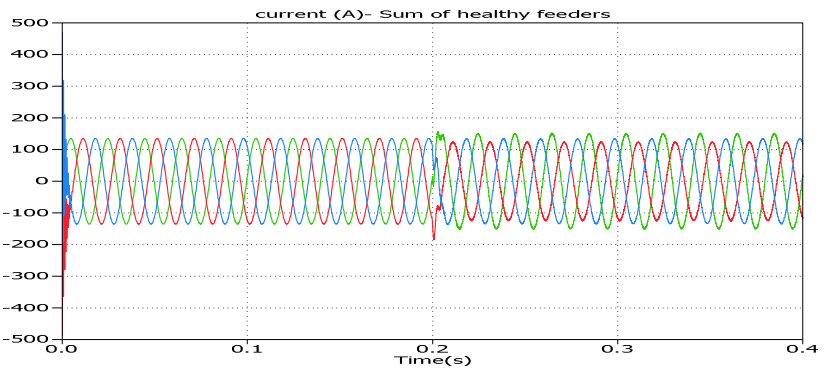

(c)

Fig. 7. (a) Fault current during the single-phase earth fault in power grid compensated by ASC. (b) Current of faulty feeder. (c) Summation of current of the healthy feeders. 


\section{B. Compensation using parallel operation of ASC and 4-leg transformer}

In this case, the proposed 4-leg three-phase transformer is connected to feeder 4 . The maximum magnetic flux density for this transformer is designed to $\mathrm{B}=1.3 \mathrm{~T}$ and the relative permeability is $\mu_{r}=40000$. TABLE II summarizes parameters of the proposed compensation transformer. Mathematical model including the four-leg magnetic core was built in PLECS (see Fig. 8). The fourth leg that is in parallel to other legs with winding is split by an air gap, used to adjust the zero-sequence impedance of the transformer which affects the magnitude of required compensation current. Thus, the transformer design can be adjusted to a particular distribution grid with specific parasitic capacitance.

TABLE II. PARAMETERS OF 4-LEG TRANSFORMER

\begin{tabular}{|c|c|c|}
\hline Prameter & Value & Unit \\
\hline Primary Resistance & 1.03 & $\Omega$ \\
\hline Secondary Resistance & 0.7 & $\mathrm{~m} \Omega$ \\
\hline Primary Voltage & 22 & $\mathrm{kV}$ \\
\hline Secondary Voltage & 400 & $\mathrm{~V}$ \\
\hline Primary Current & 33.3 & $\mathrm{~A}$ \\
\hline Secondary Current & 1125 & $\mathrm{~A}$ \\
\hline Primary winding turns & 1650 & - \\
\hline Secondary winding turns & 30 & - \\
\hline
\end{tabular}

During the fault, the zero-sequence voltage is present and the source of the zero-sequence voltage is the negative of the phase voltage of the distribution transformer: $V_{0}=-V_{c n}$ when the fault is in phase $\mathrm{c}$. The zero-sequence component of the flux (i.e. the flux $\varphi a+\varphi b+\varphi c$ flowing via the fourth leg) is integral of the voltage $V_{0}$ over time. Start of integration corresponds to the ignition of the fault and this affects the offset (initial dc component) of the flux.

The flux can be computed using a well-known relation:

$$
\varphi=\frac{F}{R}=\frac{N I}{R},
$$

where $F$ and $\mathrm{R}$, are ampere-turns and reluctance of the transformer respectively.

Equation (6) represents the relation between magnetic flux density and area of the magnetic core, from which the area is calculated:

$$
\varphi=B A .
$$

The reluctance of the air gap in the fourth leg is calculated as

$$
R=\frac{l}{\mu_{0} A},
$$

where $l$ and $A$ are length and area of the air gap respectively and the permeability of the air is $\mu_{0}=4 \pi \times$ $10^{-7}$.

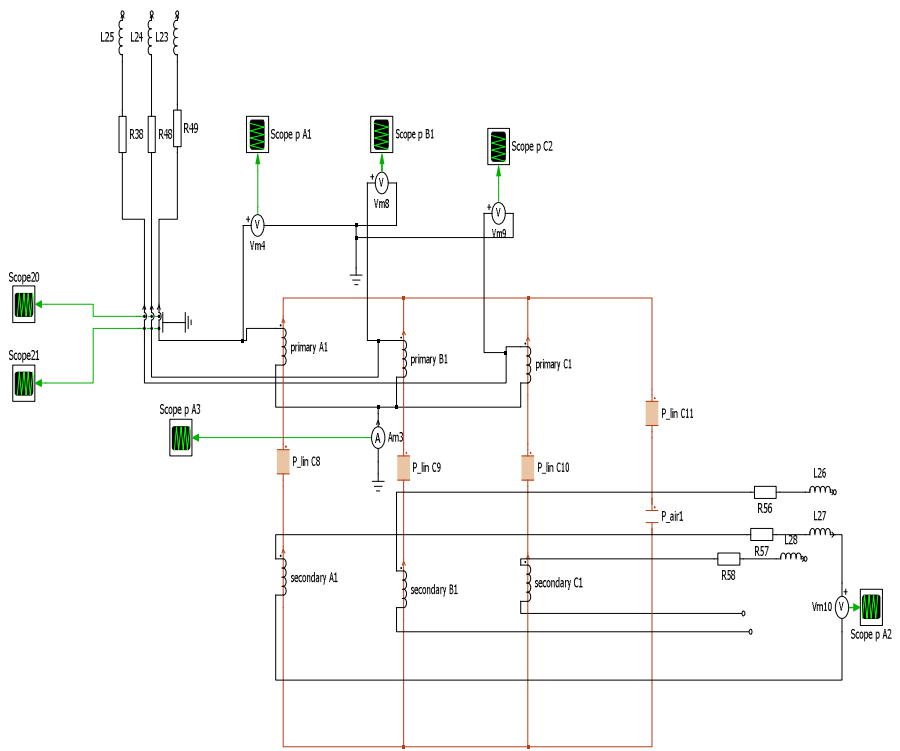

Fig. 8. Mathematical model of proposed 4-leg transformer in PLECS

Regarding equation (4), the flux of each phase, could be calculated which is $0.06 \mathrm{~Wb}$. The flux of the fourth leg of the transformer is equal to the sum of the fluxes of the other phases, which is equal to $0.12 \mathrm{~Wb}$.

When single-phase earth fault occurs, there is in a maximum zero-sequence voltage of $12.7 \mathrm{kV}(\mathrm{rms})$ on the transformer. In this state, transformer has to generate the compensation current 33.3 A (zero-sequence component, flowing to the earth point). From that knowledge, we can compute the reactance $\left(X_{0}=\omega L_{0}\right)$ for the zero-sequence component: $X_{0}=\omega L_{0}=\frac{U_{0}}{I_{0}}=\frac{12700}{33.3}=381.38$,

$$
L_{0}=\frac{X_{0}}{\omega}=\frac{381.38}{2 \pi \times 50}=1.21 \mathrm{H},
$$

Thus, the transformer's inductance for the zero-sequence component is $1.21 \mathrm{H}$.

According to Equation (6), the area of the fourth leg of the transformer can be calculated, which is $0.0923 \mathrm{~m}^{2}$. The neutral current of 4-leg transformer and ASC with zero sequence of primary side of 4-leg transformer are shown in Fig.9.

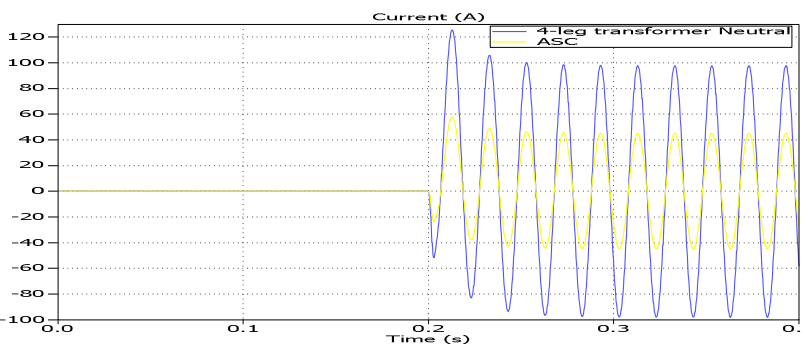

(a)

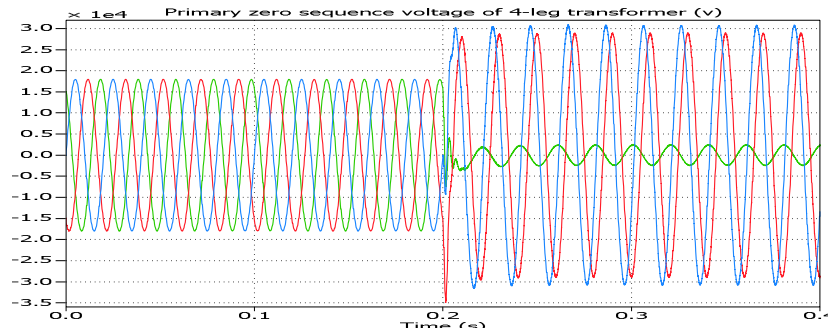


(b)

Fig. 9. (a) Current of neutral of 4-leg transformer and ASC. (b) Zero sequence voltage of 4-leg transformer (primary side).

By using the equation (5), the value of air gap reluctance will be obtained, and as respects the reluctance of air gap is much more than the core reluctance in the fourth leg, so with good approximation only air gap reluctance considered to be. Finally, using equation (7), the length of the air gap can be obtained; its final value is $0.053 \mathrm{~m}$.

In this case, the compensation current of the 4-leg transformer is $100 \mathrm{~A}$ and ASC's current is $40 \mathrm{~A}$. The primary currents, zero sequence voltages and summation of primary currents of the 4-leg transformer are depicted in Fig.10. They match each other (it corresponds to the zero-sequence component) and their summation in the neutral point of the star-connected primary winding results in the total compensation current of the transformer.

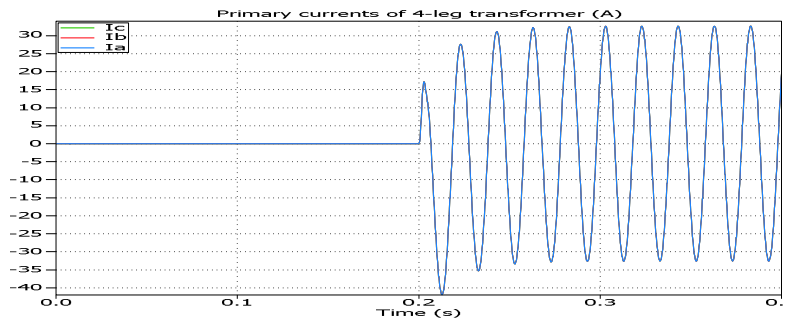

(a)

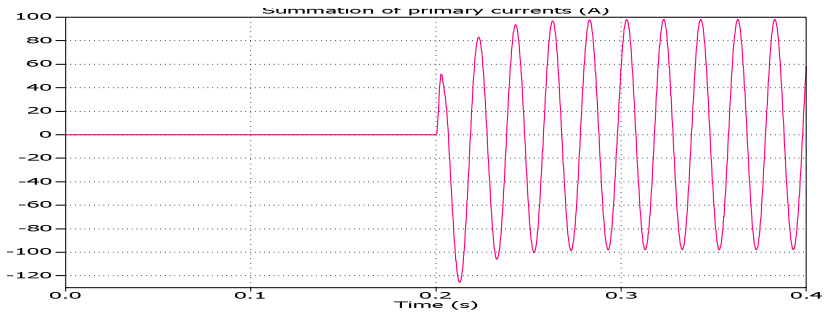

(b)

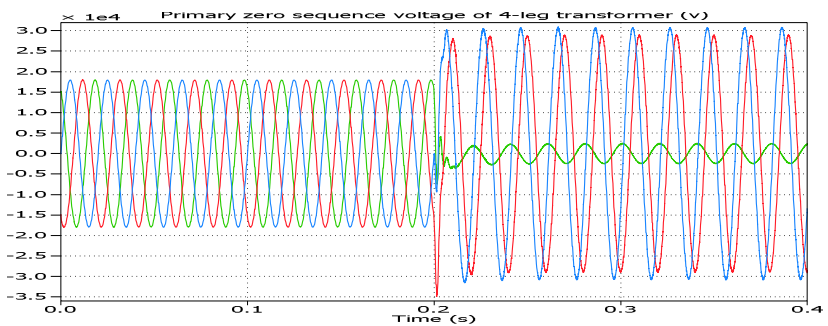

(c)

Fig. 10. (a) Primary currents of 4-leg transformer. (b) Summation of primary currents of 4-leg transformer. (c) Primary zero sequence voltages of 4-leg transformer.

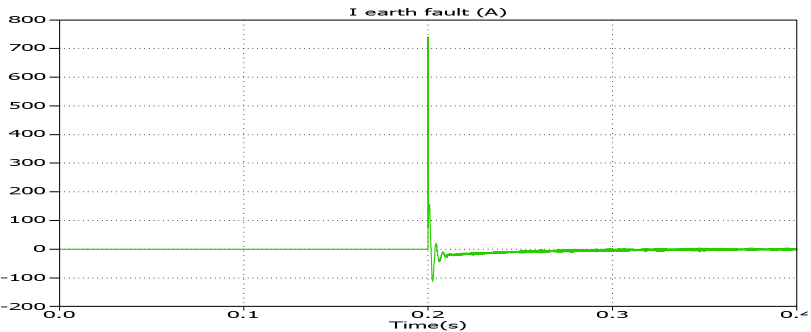

(a)

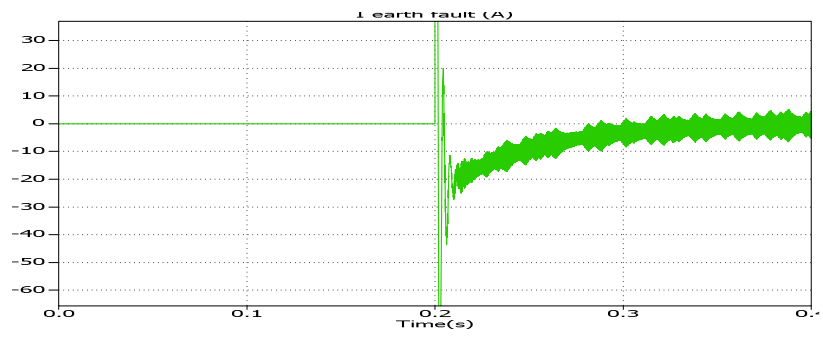

(b)

Fig. 11. (a) Peak current of single-phase earth fault compensated by cooperation of ASC and 4-leg transformer. (b) steady state of earth fault

The single-phase earth fault current is shown in Fig.11. It is reduced to below $5 \mathrm{~A}$. In this case, the compensation current is equal to summation of 4-leg transformer and $\mathrm{ASC}$, that means the 4-leg transformer can help the ASC for compensating the earth fault. One of the advantages of this transformer is that it can be connected anywhere in the network, and as the number of consumers increases, the network becomes larger, and this transformer can help ASC for clearing the fault current.

\section{Network compensated by standalone 4-leg transformer}

In this section, the ASC is opened and the 4-leg transformer alone eliminates the earth fault. The earth fault current, zero sequence voltage, primary currents and summation of primary currents of 4-leg transformer are shown on Fig.12. As can be seen, without the ASC, the 4-leg transformer's compensation performance corresponds to the ASC. Without the ASC, the total compensation current is equal to the total zero-sequence current of the transformer, generated due to the zero-sequence voltage present during the fault.

Moreover, a voltage source can be connected to the secondary side of the transformer by which the zero-sequence component of the current can be controlled, and thus, the compensation performance can be improved. To control the primary current of the 4-leg transformer, the secondary can be connected to a power converter or an adjustable inductor can

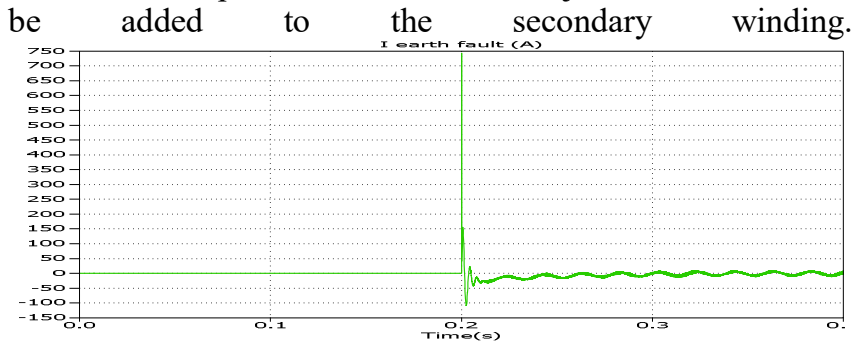

(a)

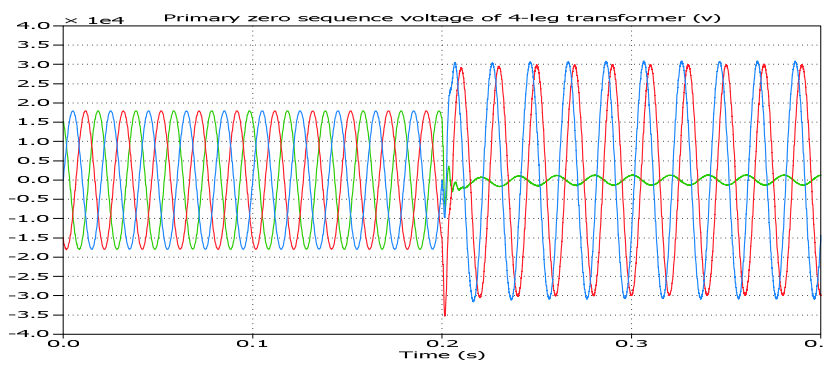




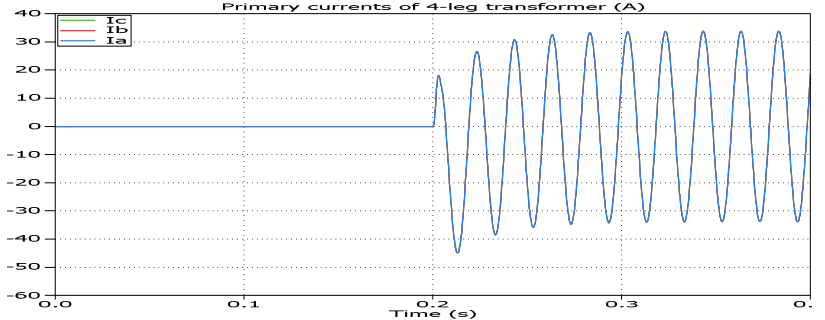

(c)

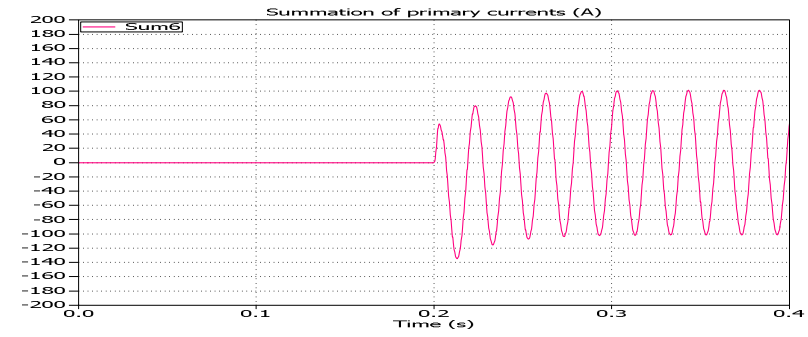

(d)

Fig. 12. (a) Fault current. (b) Zero sequence voltage. (c) Primary currents.

(d) Summation of primary currents, when compensated by standalone 4-leg transformer

With comparing the single earth fault currents in three modes it can be concluded that the performance of the transformer corresponds to that of the ASC. It can generate the zero-sequence current compensating the reactive part of the fault current and the 4-leg transformer can provide a full compensation of the grid in the standalone operation mode, and it can assist to the ASC and provide part of the required total compensation current. Since it is connected to phase conductors, it can be installed anywhere in the grid. Thus, it is suitable for distributed compensation. It is also suitable for grids where the neutral of the distribution transformer is not available. With these explanation, transformer can be used instead of Petersen coil, as the network gets larger, the transformer can eliminate single-phase fault current and be installed anywhere in the network, and it is easier to adjust than the Petersen coil, while the Petersen coil can't be installed anywhere. One of the important benefit of this method is adjusting the zero sequence current for single phase-earth fault.

\section{CONCLUSION}

A new device to reduction of the single-phase earth fault current is proposed in the paper. The compensation is achieved by controlling the zero-sequence current flowing through the proposed 4-leg transformer which is connected to phase conductors of the network. Using a special design employing a four-leg magnetic core with an air gap of designed reluctance, the zero-sequence impedance of the transformer can be controlled and thus, the required compensation current can be generated, if the zero-sequence voltage is present. One of the benefits is that the transformer can be connected anywhere in the network and it is suitable for a distributed compensation. Theoretical assumptions have been verified by simulations performed on a mathematical model in the PLECS software. The results have confirmed that the performance of the proposed 4-leg transformer corresponds to the ASC in the compensation of single-phase earth faults.
[1] H. Ricardo and T. Alexandre, "Ground fault protect ion methods for distribution system”, Art igo Tecnico, 2017, pp. 15-20.

[2] S. Ravlić, A. Marušić, "Simulation Models for Various Neutral Earthing Methods in Medium Voltage Systems", Procedia Engineering 100, 2015, pp.1182-1191.

[3] B. Gustavsen and J. A. Walseth, "A case of abnormal overvoltages in a Peterson grounded 132-kV system caused by broken conductor", IEEE Trans. Power Del., 2003, vol. 18, no. 1, pp. 195-200.

[4] J. Tian, Q. Chen, L. Cheng, and Y. Zhang, "Arc-suppression coil based on transformer with controlled load", IET Elect. Power Appl., 2010, vol. 5, no. 8, pp. 644-653.

[5] R. Burgess and A. Ahfock, "Minimising the risk of cross-country faults in systems using arc suppression coils," IET Gener., Transm. Distrib., 2011, vol. 5, no. 7, pp. 703-711.

[6] K. J. Sagastabeitia, I. Zamora, A. J. Mazon, Z. Aginako, and G. Buigues, "Phase asymmetry: A new parameter for detecting singlephase earth faults in compensated MV networks," IEEE Trans. Power Del., 2011, vol. 26, no. 4, pp. 2251-2258.

[7] F. Ibrahim Jabbar, A. Mohamad Adieb Kadhim, and A. Ahmed Abed, "Detection of Earth Fault by controlling Petersen Coil using Adaptive Neural Fuzzy Inference System in Distribution Grid", International Journal of Applied Engineering Research., 2018, vol. 13, no. 9, pp. 7006-7009.

[8] P. Vancata, I. Matuljak, "New Method of ASC Tuning Using Truly Multifrequency Current Signal," 25th International Conference on Electricity Distribution., 2019, no. 402, pp. 1-5.

[9] M. Steglich, C. Löwe, B. Bauernschmitt, C. Rehtanz, R. Böhm, J. Franke, "A novel method to reduce the harmonic currentsin the residual earth fault current during a single phase to ground fault in compensated grids,” IEEE PES Innovative Smart Grid Technologies Europe (ISGTEurope). 2019 , pp. 1-5.

[10] R. R. Karymov and M. Ebadian, "Comparison of magnetically controlled reactor (MCR) and thyristor controlled reactor (TCR) from harmonics point of view,” Int. J. Elect. Power Energy Syst., 2007, vol. 29, no. 3, pp. 191-198.

[11] S. Myint, W. Wichakool and P. Santiprapan, "A Simple High Impedance Fault Detection Method based on Phase Displacement and Zero Sequence Current for Grounded Distribution Systems," 2018 IEEE PES Asia-Pacific Power and Energy Engineering Conference (APPEEC), 2018, pp. 118-122.

[12] J. Tang, B. Xiong, Y. Li, C. Yuan and Y. Qiu, "Faulted Feeder Identification Based on Active Adjustment of Arc Suppression Coil and Similarity Measure of Zero-Sequence Currents," in IEEE Transactions on Power Delivery, 2021, pp. 1-5.

[13] A. Barili, A. Brambilla, G. Cottafava, and E. Dallago, "A simulation model for the saturable reactor," IEEE Trans. Ind. Electron., 1988, vol. 35, no. 2, pp. 301-306.

[14] N. Ning, X. Li, J. Fan, W. Ng, Y. Xu, X. Qian, and H. Seet, “A tunable magnetic inductor,” IEEE Trans. Magn., 2006, vol. 42, no. 5, pp. 15851590 .

[15] A. Farughian, L. Kumpulainen, K. Kauhaniemi "Review of methodologies for earth fault indication and location in compensated and unearthed MV distribution networks," Electric Power Systems Research., 2018, no. 154, pp. 373-380.

[16] P. Liu, C. Huang "Detecting Single-Phase-to-Ground Fault Event and Identifying Faulty Feeder in Neutral Ineffectively Grounded Distribution System," IEEE TRANSACTIONS ON POWER DELIVERY, VOL. 33, NO. 5, 2018, pp 2265-2273.

[17] D. Topolanek ; M. Lehtonen ; P. Toman; J. Orsagova; J. Drapela “An earth fault location method based on negative sequence voltage changes at low voltage side of distribution transformers", Electrical Power and Energy Systems, 2020, pp 1-8.

[18] M. Ojaghi, J. Faiz, M. Kazemi, M. Rezaei "Performance Analysis of Saturated Induction Motors by Virtual Tests," IEEE TRANSACTIONS ON EDUCATION, VOL. 55, NO. 3, 2012. pp 370377.

[19] M.Odonovan, E.Cowhey, N. Barry, "Implentation of Intelligent Tuning System for Petersen Coils on the Irish Distribution Network," 53rd International Universities Power Engineering Conference (UPEC), 2018, pp 1-6. 